\title{
Permanente Kampagnenforschung
}

Holtz-Bacha, Christina (Hrsg.): Die Massenmedien im Wahlkampf. Die Bundestagswahl 2005, VS Verlag für Sozialwissenschaften, Wiesbaden 2006, 360 Seiten, € 34,90.

Maurer, Marcus, Carsten Reinemann, Jürgen Maier und Michaela Maier: Schröder gegen Merkel. Wahrnehmung und Wirkung des TV-Duells 2005 im Ost-West-Vergleich, VS Verlag für Sozialwissenschaften, Wiesbaden 2007, 258 Seiten, € 24,90.

Wagner, Jochen W.: Deutsche Wahlwerbekampagnen made in USA? Amerikanisierung oder Modernisierung bundesrepublikanischer Wablkampagnen, VS Verlag für Sozialwissenschaften, Wiesbaden 2005, 462 Seiten, $€ 49,90$.

In Deutschland wird eigentlich immer irgendwo gerade ein Wahlkampf geführt, dessen Ergebnisse bundespolitische Bedeutung haben können. Auch die Forschung dazu ist inzwischen gut etabliert. Dabei richtet sich der Blick nicht mehr allein auf die Wahlsoziologie und die sozialpsychologische Erklärung der individuellen Wahlentscheidung, sondern zunehmend auf die Frage, welche Strategien und Taktiken politische Akteure im Rahmen der Wahlkämpfe verfolgen. Dies behandeln auch die vorliegenden Sammelbände, die nach der Bundestagswahl 2005 veröffentlicht wurden.

Die Beiträge im Buch von Christina Holtz-Bacha gehen, anders als man den Titel leicht verstehen könnte, nicht allein auf die Bedeutung journalistischer Berichterstattung für Wahlkämpfe ein, denn es wird ein breiter Medienbegriff angelegt: Ausführlich beschäftigen sich die Verfasser mit den Wahlplakaten, Printanzeigen, Fernsehspots, Homepages und Blogs im Internet. Die Beiträge greifen meist aus kommunikationswissenschaftlicher Perspektive ihr jeweiliges Thema auf und sind durchgängig gut lesbar und praxisorientiert geschrieben.

Mit dem Einstiegskapitel wird eine Zusammenfassung über den Verlauf der Kampagnen und die Ergebnisse der Bundestagswahl 2005 gegeben, die ja aufgrund der (absichtsvoll) gescheiterten Vertrauensfrage des Bundeskanzlers Gerhard Schröder überraschend früh stattfand. Deutlich verkürzt war daher die Zeit des Wahlkampfes selbst, aber insbesondere auch dessen Vorbereitung und Planung musste schneller ablaufen als sonst. Die verfügbaren finanziellen Ressourcen der Parteien waren dadurch erheblich geschmälert.

Detailliert zeichnen Christina Holtz-Bacha und Eva-Maria Lessinger den Plakatwahlkampf nach, der in Deutschland nach wie vor eine besonders große Rolle spielt. Hier werden echte Primärdaten berichtet, von den Namen der eingesetzten Werbeagenturen bis zur Zahl der jeweils aufgestellten Plakate. Daran schließt sich eine „ikonographischikonologische“ Untersuchung an (S. 91). Insgesamt stellen die Verfasserinnen für die CDU eine "gewisse Beliebigkeit beim Plakatauftritt" und ein fehlendes Corporate Image fest (S. 122). Den anderen Parteien wird jeweils professionelle Einheitlichkeit in der Darstellung attestiert, die im Jahr 2005 jedoch wenig Innovatives enthielt. Ein umfassender Vergleich der Plakatkampagnen miteinander und mit den Vorjahren findet nicht statt; es wird lediglich darauf hingewiesen, dass 2005 aufwändigere Plakate im Reportage-Stil oder humoristische Ansätze nicht genutzt wurden. Stattdessen kamen vor allem die klassischen Kandidatenportraits und - gerade bei der SPD - sehr textlastige Motive zum Einsatz. Die Vermutung, dass dies auch in der knappen für die Wahlkampfvorbereitung verfügbaren Zeit begründet lag (S. 124), ist sehr plausibel. Darauf führen die Verfasserinnen in einem weiteren Beitrag übrigens auch die „allgemeine Lustlosigkeit bei der Spot-Gestaltung“ in der Fernsehwerbung zurück (S. 181). 
Ähnlich ausführlich beleuchtet Sandra Lieske die Anzeigenkampagnen in den Printmedien, indem sie die Frankfurter Allgemeine Zeitung, Süddeutsche Zeitung, Bild-Zeitung und das Nachrichtenmagazin Der Spiegel auswertet. Zusätzlich hat sie die Parteien befragt wobei sich herausstellte, dass diese zur gezielten Ansprache von Wählerinnen und Wählern insbesondere in stärker spezialisierten Zeitschriften und regionalen Zeitungen inserierten. Inwiefern daher die ausgewerteten 14 Anzeigen in der überregionalen Presse wirklich ein adäquates Gesamtbild bieten können, ist fraglich. Schon rein äußerlich wird deutlich, dass sie vielfach den Wahlplakaten ähnlich sind, und auch in der Redaktion des Sammelbandes ist dies offenbar durcheinandergeraten: Die Abbildungsbeschriftungen bezeichnen alle Anzeigen als "Wahlplakate“ (S. 136 bis 152).

Eine methodisch ausgereifte inhaltsanalytische Untersuchung des Online-Wahlkampfes legt Eva Johanna Schweitzer vor. Für die im Bundestag vertretenen Parteien stellt sie dabei im Vergleich zu den Vorjahren eine deutliche formale Professionalisierung fest, sieht aber bei kleineren Parteien durchgängig „erhebliche Defizite“ (S. 197, 199). Während insgesamt mehr Wert auf den Online-Wahlkampf gelegt wurde und dort mehr „Information, Interaktivität und Serviceorientierung" gezeigt wird als zuvor (S. 209), kann eine stärkere Personalisierung oder Negativität im Internetwahlkampf der Parteien nicht nachgewiesen werden: Trotz der Bedeutung der Spitzenkandidaten und insbesondere auch der Diskussion um das Steuerkonzept von Paul Kirchhof im Wahlkampf 2005 bezogen sich über drei Viertel aller politischen Aussagen auf die Parteien und Fraktionen und nicht auf Personen (S. 204).

Beim Blick auf die Fernsehberichterstattung zur Bundestagswahl stellen Winfried Schulz und Reimar Zeh eine im Vergleich zur Wahl 2002 erhöhte journalistische Beachtung der Wahlkampfstrategien und -taktiken fest, während die Kandidatenorientierung rückläufig gewesen sei (S. 284 f.). Vorherrschend bleibe weiterhin der Blick auf die politischen Prozesse, die so genannte Politics-Dimension (S. 290). Diese Erkenntnisse passen zu der im englischsprachigen Raum verbreiteten Einschätzung, dass über Wahlkämpfe zunehmend so wie über Pferderennen berichtet werde („Horse Race Coverage“): Fast minütlich wird festgehalten, wer vorne liegt, mit welchen Winkelzügen dies erreicht wurde und was die nächsten Schritte sein könnten - oder sollten. Nicht nachzuweisen war hingegen ein besonderer Bonus für den Amtsinhaber (S. 293 f.), und die erste weibliche Kandidatin für das Amt des Bundeskanzlers sah sich keinen nennenswerten klischeehaften Urteilen ausgesetzt (S. 301).

Ebenso gründlich wie das Fernsehen werten Jürgen Wilke und Carsten Reinemann die Presseberichterstattung aus. Sie stellen hier eine starke Personalisierung fest, sprechen von einer „Explosion visueller Darstellungen der Kandidaten“ (S. 335) und einem Hang zur negativen Sichtweise. Auch im Printbereich ist kein Kanzlerbonus zu beobachten.

In Stil und redaktioneller Qualität aus dem (wissenschaftlichen) Rahmen des Sammelbandes fällt der Beitrag von Kerstin Plehwe, laut Autorenverzeichnis „einer der führenden Köpfe im Bereich der Dialogkommunikation“ (S. 358). Der ohnehin kurze Beitrag wirkt wie aus verschiedenen Vorträgen zusammengestellt; das Fazit nimmt eine Aufzählungsliste mit direkten Appellen an die Leserschaft auf, verwendete Begriffe bleiben unscharf, und es mangelt an Belegen und Quellenangaben. Die Aussage, dass in Großbritannien ein „System der direkten Demokratie“ herrsche (S. 242) entspricht ebenso wenig der Realität wie die Andeutung, dass deutschen Parlamentariern die speziellen Probleme ihres jeweiligen Wahlkreises weitgehend egal sein könnten (ebenda).

Besonders lesenswert ist hingegen der zum Einstieg in das Buch prominent platzierte Beitrag von Thomas Bosch, der sich aus Sicht der SPD mit der Mobilisierung der eigenen Mit- 
glieder beschäftigt. So wird - im angemessenen Gegensatz zum Titel des Sammelbandes darauf hingewiesen, dass auch im Medienzeitalter die Parteimitglieder weiter eine wichtige Rolle für den Wahlkampf spielen. Bei aller zentralen Strategiefindung, externen Unterstützung durch Werbeagenturen und professioneller Maßnahmenplanung, bleiben sie es, die „vor Ort auf lokaler Ebene“ diese Vorhaben umsetzen und realisieren (S. 35). Thomas Bosch, selbst beim Parteivorstand der SPD tätig, berichtet von der zentralen Herausforderung, „Mitglieder zu mobilisieren, die sich größtenteils eigentlich gar nicht mobilisieren lassen wollen“ (S. 41), da 2005 in der Partei zeitweise eine „nahezu apokalyptische Untergangsstimmung" (S. 40) geherrscht habe. Gelungen sei es insbesondere mit Hilfe des motivierenden Einsatzes von Gerhard Schröder sowie der speziellen Form der ,zentralisierten Dezentralisierung" (S. 55), bei der die Wahlkampagne in der Parteizentrale mit 250 Mitarbeitern sich auf die Koordination und Planung konzentrierte und sich als Dienstleister für die Aktiven vor Ort positionierte. Die abschließende These, dass die Zeit reiner Medienwahlkämpfe vorbei sei und die Parteimitglieder eine Renaissance erlebt hätten (S. 76), gilt es künftig in der Politikwissenschaft zu beachten, aber auch vor dem Hintergrund der weiter rückläufigen Mitgliedschaften zu hinterfragen.

Insgesamt weisen die Beiträge des Bandes einen eher geringen Theoriebezug auf und sind wenig analysierend verfasst; doch in der detaillierten, wohlgeordneten und wissenschaftlich durchweg sorgfältigen Deskription liegt ihre besondere Stärke.

Enthalten ist dort auch eine Untersuchung zu einem jungen Element deutscher Wahlkämpfe: der formalisierten Fernsehdiskussion zwischen den Spitzenkandidaten, die in Deutschland gerne dramatisierend als „Duell“ bezeichnet wird. Christoph Tapper und Thorsten Quandt werten detailliert die Gesprächsführung und Inhalte der Debatte zwischen Angela Merkel und Gerhard Schröder aus. Wenngleich die Verfasser ausdrücklich auf die vorherrschend moderierende Rolle der beteiligten Journalisten und der vorher detailliert ausgehandelten Regeln eingehen, bleibt doch der Eindruck einer sehr stark durch den Blick auf die Persönlichkeiten der beiden Kandidaten beeinflussten Sichtweise dieses Berichtes.

Zum vertieften Verständnis solcher „Duelle“, die aller Voraussicht auch in Zukunft fester Teil deutscher Wahlkämpfe bleiben, lohnt ein Blick in den Band von Marcus Maurer, Carsten Reinemann, Jürgen Maier und Michaela Maier. Er erhellt nicht nur die Debatten in ihrem Verlauf ausführlich, sondern berücksichtigt zudem die unmittelbare Publikumswahrnehmung, die Medienberichterstattung sowie deren Wirkung auf die Zuschauer. Damit folgen sie einem gut nachvollziehbaren und umfassenden Design, das an langjährige Erfahrungen und Erkenntnisse aus der etablierten U.S.-amerikanischen Debattenforschung anknüpft.

Wesentlich stützt sich die Untersuchung der Publikumswirkung auf die so genannte „Real-Time-Response-Messung (RTR)“, bei der eine Gruppe von Zuschauern mit Hilfe von speziellen Geräten laufend ihre Einschätzung abgibt. Im Präsidentschaftswahlkampf 2008 strahlte der Nachrichtensender CNN die Ergebnisse solcher Befragungen sogar live während der Debatten aus. Die Studie für das deutsche TV-Duell 2005 wurde parallel in Mainz und Jena durchgeführt, um Ost- und Westdeutschland zu berücksichtigen. Es beteiligten sich 72 und 49 Personen, die zusätzlich unmittelbar vor und nach der Debatte sowie nochmals vier Tage später mit Hilfe eines standardisierten Fragebogens interviewt wurden. Wenngleich auf eine ausgewogene und regional angepasste Verteilung sozioökonomischer Merkmale und politischer Einstellungen Wert gelegt wurde, war die Gruppe nicht repräsentativ; darauf wird im methodischen Teil hingewiesen (S. 26 f.), doch bei der Interpreta- 
tion der Ergebnisse sollte man sich dies öfter in Erinnerung rufen, gerade wenn über Untergruppen berichtet wird, zum Beispiel „die Jenaer Ungebundenen“ (S. 76), die aus gerade noch 27 Personen bestehen, oder aber auch statistisch nicht signifikante Ergebnisse als Tendenzaussagen dargestellt werden (zum Beispiel S. 57, 102 und 138).

Inwiefern Fernsehdebatten eine Wirkung auf Wissen und Verhalten der Wählerschaft haben, ist eine der häufigen politikwissenschaftlichen Fragen. Abschließende Antworten dazu kann die Studie allerdings auch aufgrund der kleinen Fallzahlen kaum geben; die Verfasser sehen eine Steigerung von inhaltlichem Wissen insbesondere bei politisch schwächer interessierten Zuschauern, während bei stärker interessierten die parteipolitisch selektive Wahrnehmung von Aussagen auch zu „Wissensverlusten“ geführt habe (S. 142). Einige Wähler änderten ihre geäußerte Kanzlerpräferenz durchaus im Anschluss an die Debatte oder wandten sich einem Kandidaten erstmals zu (S. 108). Gründlich untersucht werden weiterhin die Wirkung der Debatten auf die Urteilskriterien der Wähler (in der Medienwirkungsforschung als „Priming“ bezeichnet), der direkte Einfluss auf ihre Wahlabsichten und auf das politische Wissen allgemein, die Inhalte und Bedeutung der Nachberichterstattung.

In Bezug auf die TV-Duelle waren viele Journalisten „vor allem Akteure - und weniger Chronisten“ (S. 192): Ihre Einschätzung unterschied sich deutlich von der Bewertung in der Bevölkerung, ihre Meinungen verbreiteten sie nicht selten mit besonderem Nachdruck, und sie unterschätzten den Neuigkeitswert der politischen Inhalte für die Bevölkerung. Insbesondere kritisieren die Verfasser den Umgang mit Blitzumfragen (S. 193). Dieses Kapitel und die darin enthaltene deutliche Kritik sei den Medienschaffenden zur Vorbereitung einer möglichen Debatte im Jahr 2009 dringend empfohlen.

In ihrem Schlusskapitel räumen die Verfasser noch einmal mit vielen Vorurteilen auf: Zwar konzentrierten sich die beiden Kandidaten bei ihren Aussagen auf emotionale Appelle und die Äußerung von Gemeinplätzen, und die Zuschauer nahmen diese Veranstaltung „durch ihre parteipolitische Brille“wahr (S. 232). Doch deutlich wurde auch, dass die visuellen Eindrücke für die Bewertung der Zuschauer weit weniger relevant sind als die inhaltlichen Aussagen (S. 233). Während dieses Sendeformat wohl die Personalisierung der Wahlentscheidung auch befördere, führe sie doch zugleich zu einem Wissenszuwachs über die inhaltlichen Positionen der Parteien und Kandidaten (S. 236).

Nicht erst anlässlich dieser Fernsehdebatten wird in Medien und Wissenschaft seit Jahren regelmäßig darüber diskutiert, ob man allgemein von einer Amerikanisierung der deutschen Wahlkampfführung sprechen kann. Viele der dazu erschienenen Beiträge übertreiben entsprechende Entwicklungen und vernachlässigen relevante Rahmenbedingungen. Zu diesem Thema hat Jochen W. Wagner bereits im Jahr 2005 eine fundierte Untersuchung der Wahlkampagnen in Deutschland und den USA im Vergleich vorgelegt. Während die sehr ausführliche Darstellung des theoretischen Bezugsrahmens eine stärkere Fokussierung und klarere Strukturierung vertragen hätte, zeichnen sich die Überblicksdarstellungen zu beiden politischen Systemen durch eine klare Konzentration auf das Wesentliche aus. Einige Unschärfen verwundern: So wird etwa der Beginn des „Going Public“ des U.S.-Präsidenten auf die Regierungszeit Richard Nixons datiert (S. 108), anstatt die deutlich größere Bedeutung sowohl von Franklin D. Roosevelt und John F. Kennedy zu würdigen; „logrolling“ - die informelle gegenseitige Unterstützung von Gesetzesvorhaben durch den zeitlich verschobenen Austausch von Stimmen im Kongress - wird als „Schneeballprinzip“ übersetzt (S. 115), und die Darstellung der öffentlichen Kampagnenfinanzierung bringt die Regelungen der Haupt- und Vorwahlen durcheinander (S. 121). Für Deutschland wird fälschlicherweise 
formuliert, dass ein Bundeskanzler Mitglied des Bundestages sein müsse (S. 142), und auch die Behauptung, in deutschen Wahlkämpfen herrsche „de facto ein Zweiparteiensystem“, führt in die Irre (S. 143).

Die Stärke der Studie liegt jedoch in der konzisen Kurzdarstellung der Wahlkämpfe der vergangenen 50 Jahre in Deutschland und den USA, die sich auf umfassende Quellenarbeit stützt und entsprechende Verweise auf das fast 50-seitige Literaturverzeichnis beinhaltet. Überdies wurden zahlreiche Experteninterviews mit Politikberatern und wissenschaftlichen Beobachtern geführt, die in einer teilstandardisierten Form ausgewertet wurden.

Im Ergebnis hält Jochen W. Wagner fest, was in den vergangenen Jahren schon mehrfach formuliert wurde: Der Amerikanisierungs-Begriff ist den tatsächlichen Entwicklungen nicht angemessen. Viele der als neu wahrgenommenen Elemente moderner Wahlkampfführung (zum Beispiel Personalisierung, Zielgruppenansprache, Einsatz von Beratern, Nutzung von Demoskopie) sind nicht selten schon seit den 1950er Jahren in Deutschland etabliert. Ebenso lange beobachten die deutschen Parteizentralen intensiv amerikanische Wahlkämpfe, wobei keine blinde Übernahme von Techniken oder Strategien stattfindet: „Es zeigt sich vielmehr, dass eine eigenständige deutsche Kampagnenkultur besteht, die durch mehr als 50 Jahre Praxis so weit gefestigt ist, dass sie bedarfsorientiert und aus eigenem Antrieb selektiv Transferoptionen nutzen kann.“ (S. 405)

Die Zusammenschau der genannten Bücher macht deutlich: Wahlkämpfe entwickeln sich ausgesprochen dynamisch mit der verfügbaren Technologie, aber auch in Abhängigkeit von den politischen Rahmenbedingungen. Durchaus dienen dabei den Kandidaten, Parteien oder Medien auch internationale Erfahrungen als Inspiration. Bedingungen erfolgreicher Wahlkampfführung und die Diffusion von verschiedenen Innovationen in angemessener Komplexität zu erhellen, bleibt eine bedeutsame Herausforderung an Politik- und Kommunikationswissenschaft. Nur gut, dass das politische System Deutschlands auch weiterhin die Möglichkeit bietet, fast permanent Kampagnenforschung zu betreiben - denn irgendwo wird ja immer gewählt.

Sven T. Siefken

\section{Standardwerk der qualitativen Nichtwählerforschung}

Steinbrecher, Markus, Sandra Huber und Hans Rattinger: Turnout in Germany. Citizen Participation in State, Federal, and European Elections since 1979 (Studien zur Wahl-und Einstellungsforschung, Band 1), Nomos Verlagsgesellschaft, Baden-Baden 2007, 342 Seiten, € 49,-.

Nachdem sich der Fokus der deutschen Politikwissenschaft in den 1990er Jahren verstärkt auf die Wahlbeteiligung gerichtet hatte, begründet durch deren stetiges Absinken seit Ende der 1970er Jahre, wurde es erst einmal ruhiger auf diesem Gebiet. Der vorliegende Band ist daher eine längst überfällige Studie zur Erforschung von Wahlbeteiligung und individueller Wahlabstinenz in Deutschland. Sie zeichnet sich durch eine systematische und theoriegeleitete Vorgehensweise und die Nutzung umfangreichen empirischen Materials aus, das sowohl Bundestags-, Landtags- als auch Europawahlen berücksichtigt. Dadurch gelingt es den Autoren, den Stand der deutschen Nichtwählerforschung zu aktualisieren und zu erweitern. Ihr wesentliches Verdienst besteht in der akribischen Begehung nahezu aller bekannten Wege der Wahlforschung, um die Motive der Nichtwähler, aber auch die räumliche und zeitliche Struktur der Wahlbeteiligung zu ergründen. Lediglich der qualitative Pfad bleibt unbeschritten. 